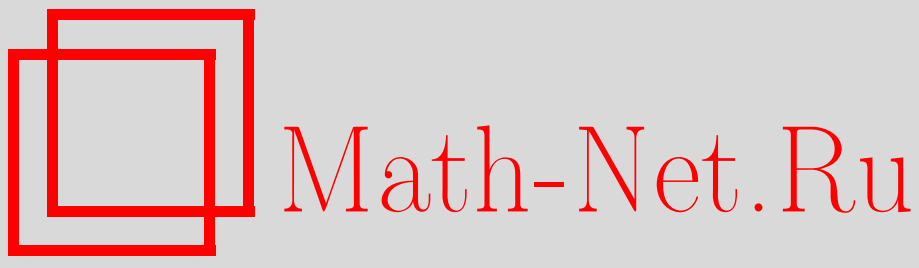

А. И. Штерн, Критерии непрерывности конечномерных представлений связных локально компактных групп, Матем. сб., 2004, том 195, номер 9, 145-159

DOI: https://doi.org/10.4213/sm849

Использование Общероссийского математического портала Math-Net.Ru подразумевает, что вы прочитали и согласны с пользовательским соглашением

http://www . mathnet.ru/rus/agreement

Параметры загрузки:

IP : 44.207 .124 .84

26 апреля 2023 г., 05:23:35 
УДК $512.546+517.987$

\title{
А.И.Штерн
}

\section{Критерии непрерывности конечномерных представлений связных локально компактных групп}

\begin{abstract}
Получены необходимые и достаточные условия непрерьвности конечномерных (алгебраических) представлений связных локально компактных групп. А именно показано, что конечномерное представление связной локально компактной группы непрерьвно в том и только том случае, если колебание этого представления в единице групшы меньше 2 .
\end{abstract}

Библиографоия: 12 названий.

\section{§1. Введение}

Условия непрерывности гомоморфизмов и представлений топологических групп и полугрупп изучались, как правило, в предположении измеримости в том или ином смысле рассматриваемых отображений. Однако условие измеримости отображения является ограничительным, и проверка этого условия не всегда проста [1]. В связи с этим представляют интерес условия непрерывности на основе оценки величины сильной вариации в точке. Изучение этих условий начато в [1], где постановка задачи мотивируется и подробно обсуждается (см. также [2]-[4]). В частности, известен соответствующий критерий непрерывности представлений локально компактных групп ограниченными линейными операторами в банаховом пространстве. Чтобы сформулировать его, напомним основное определение.

ОПрЕДЕЛЕНИЕ 1. Если $G$ - топологическая группа, $S$ - ее (алгебраическое) представление в нормированном пространстве $E$, а $E^{*}$ - пространство, сопряженное к $E$, то слабая вариачия $\omega(S) \geqslant 0$ представления $S$ в единичном әлементе е группы $G$ (или, кратко, слабая вариация представления $S$ ) определяется как верхняя грань по всем векторам $\xi \in E$ единичной длины и всем функционалам $f \in E^{*}$ единичной нормы от нижних граней величин $\sup _{g \in U}|f(S(g) \xi-\xi)|$ по всем окрестностям $U \subset G$ единичного элемента $e \in G$,

$$
\omega(S)=\sup _{\substack{\xi \in E,\|\xi\| \leqslant 1 \\ f \in E^{*},\|f\| \leqslant 1}} \inf _{U \ni e} \sup _{g \in U}|f(S(g) \xi-\xi)| .
$$

Положим также

$$
v(S)=\inf _{U \ni e} \sup _{g \in U}\left\|S(g)-1_{E}\right\|
$$

В терминах слабого колебания (1) общий критерий непрерывности банаховых представлений локально компактной групшы выглядит следующим образом.

Работа вьполнена при поддержке Российского фонда фундаментальных исследований (грант № 02-01-00574). 
ТЕОРема 1 [1]. Пусть $G$ - локально компактная группа, $S$ - ее (алгебраическое) представление в банаховом пространстве $E$ непрерывными линейными операторами. Следующие условия равносильны:

1) представление $S$ непрерывно в сильной операторной топологии;

2) $\omega(S)<1$.

Таким образом, если слабое колебание представления локально компактной групшы в банаховом пространстве удовлетворяет неравенству в условии 2) теоремы 1, то это колебание равно нулю и представление непрерывно.

Возникает естественный вопрос о возможности улучшения величины постоянной 1, обеспечивающей непрерывность представления. Очевидный пример унитарного представления недискретной топологической групшы $G$ сдвигами в пространстве $l^{2}(G)$ показывает, что, вообще говоря, нельзя заменить условие $\omega(S)<1$ в п. 2) теоремы 1 условием $\omega(S)<q$ при $q>1$. Как мы знаем, для конечномерных представлений общих локально компактных групп можно заменить условие $\omega(S)<1$ в п. 2) теоремы 1 условием $\omega(S)<\sqrt{3}$ (см. [1]). Мы увидим, что для представлений связных локально компактных групп в конечномерных нормированных пространствах можно увеличить постоянную $\sqrt{3}$ до 2 (ср. [3]). Напомним (см. [1]), что А. С. Мищенко высказал гипотезу, что для конечномерных представлений любой связной топологической групшы постоянная $\sqrt{3}$ может быть улучшена до 2 . Таким образом, основной результат статьи состоит в доказательстве справедливости гипотезы Мищенко для всех связных локально компактных групп. А именно справедлива следуюшая теорема (см. $\S 5)$.

ТЕОРема 7. Пусть $G$ - связная локально компактная группа и $\sigma$ - (алгебраическое) представление группь $G$ в конечномерном нормированном линейном пространстве Е. Если $\omega(\sigma)<2$, то $v(\sigma)=0$ (см. $(2))$, m.e. представление $\sigma$ непрерывно.

Этот результат имеет непосредственное следствие для отображений, близких к представлениям.

СЛЕДСТВИЕ. Пусть $G$ - связная локально компактная группа и $T$ - ее непрерывное отображсение в группу обратимых линейных операторов в конечномерном пространстве Е. Если существует такое представление $S$ группъи $G$ в $E$, что $\|S(g)-T(g)\| \leqslant C$ для всех $g \in G$ и некоторого $C, C<1$, то представление $S$ непрерывно.

Изложение ведется следуюшим образом. В $\S 2$ приведен ряд вспомогательных утверждений. В $\S 3$ основная теорема доказывается для связных локально компактных групп, а в $\S 4$ - для связных разрешимых групп. В $\S 5$ доказана основная теорема. В $\S 6$ приведены заключительные замечания и примеры.

Часть результатов статьи приведена без доказательств в [4].

\section{§2. Обозначения и вспомогательные утверждения}

Пусть $\mathbb{T}$ - одномерньй тор, $\mathbb{R}$ - поле действительных чисел, $\mathbb{C}$ - поле комплексных чисел, $\mathbb{N}$ - натуральный ряд, $\mathbb{Z}$ - аддитивная группа целых чисел. Символы $\mathbb{R}$ и $\mathbb{C}$ используются также для обозначения аддитивных групп соответствующих полей. 
Пусть $G$ - топологическая группа, $S$ - ее (алгебраическое, не обязательно непрерывное) представление в нормированном пространстве $E$ (над $\mathbb{R}$ или $\mathbb{C}$ ). Пусть $E^{*}$ - пространство, сопряженное к $E$.

ОПреДЕЛЕНИЕ 2 [1]. Введем сильную вариацию $\varepsilon(S) \geqslant 0$ представления $S$ в единичном әлементе е группь $G$ (или, кратко, сильную вариацию представления $S)$, полагая $\varepsilon(S)$ равным верхней грани по всем векторам $\xi \in E$ единичной длины от нижних граней величин

$$
\sup _{g \in U}\|S(g) \xi-\xi\|
$$

по всем окрестностям $U \subset G$ единичного элемента $e \in G$ :

$$
\varepsilon(S)=\sup _{\xi \in E,\|\xi\| \leqslant 1} \inf _{U \ni e} \sup _{g \in U}\|S(g) \xi-\xi\| .
$$

Следуюшее определение стандартно.

ОПРЕДЕЛЕНИЕ 3 . Пусть $G$ - топологическая группа, $S$ - ее (алгебраическое) представление в нормированном пространстве $E$. Мы будем говорить, что $S$ локально равномерно ограничено, если существует окрестность $V$ единичного элемента $e$ в $G$ и постоянная $C$, удовлетворяющие условию

$$
\|S(g)\| \leqslant C \text { для любого } g \in V .
$$

Напомним некоторые известные результаты.

Лемма 1 (см. [1; лемма 1]). Пусть $G$ - топологическая группа, $S-$ ее (алгебрачческое) представление в нормированном пространстве Е. Пусть $E^{*}-$ пространство, сопряженное $\kappa$ E. Пусть $\varepsilon(S), \omega(S)$ и $v(S)$ - вариачии представления $S$, введеннъе в (1)-(3).

1) Если $a \in \mathbb{R}, a>0$, то неравенство $\omega(S) \leqslant$ а выполняется тогда и только тогда, когда для любого $b>a$, любого единичного вектора $\xi$ в пространстве $E$ представления $S$ и любого функиионала $f \in E^{*}$ с единичной нормой существует такая окрестность $U=U(\xi, f) \subset G$ единичного әлемента е $\in G$, что $|f(S(g) \xi-\xi)|<b$ для всех $g \in U$. Аналогичное описание имеют величины $\varepsilon(S)$ и $v(S)$.

2) Представление $S$ непрерьвно сильно (слабо, по норме) тогда и только тогда, когда $\varepsilon(S)=0$ (соответственно $\omega(S)=0, v(S)=0)$.

3) Справедливо неравенство $\omega(S) \leqslant \varepsilon(S) \leqslant v(S)$.

4) Если величина $\varepsilon(S)$ конечна, а группа $G$ локально компактна, то представление $S$ локально равномерно ограничено. В частности, если группа $G$ компактна, то представление $S$ ограничено.

СлеДСтвИЕ 1 [1], [2]. Пусть $G$ - группа Ли, S - ее (алгебраическое) представление в конечномерном пространстве. Если представление $S$ непрерывно на каждой однопараметрической подгруппе группы $G$, то оно непреривно.

Теорема 2 [1; теорема 3]. Пусть $G$ - связная группа Ли и $S$ - (алгебраическое) представление группь $G$ в конечномерном нормированном линейном пространстве Е. Если $\omega(S)<2$, то $v(S)=0$, т.е. представление $S$ непрерьвно в сильной операторной топологии. 
Tеорема 3 [1; теорема 4]. Пусть $G$ - локально компактная группа и $S$ (алгебрачческое) представление группы $G$ в конечномерном нормированном пространстве E. Если $\varepsilon(S)<\sqrt{3}$, то $\varepsilon(S)=0$, т.е. представление $S$ непрерьвно в сильной операторной топологии.

Следуюшее утверждение показывает, что в конечномерном случае есть по сушеству только одно колебание представления в точке.

ЛЕмма 2. Пусть $G$ - локально компактная топологическая группа и $S$ представление $G$ в конечномерном нормированном пространстве $E$. Пусть $\omega(S)$ и $\varepsilon(S)$ - слабая и сильная вариации $S$ в единице группь соответственно (см. определение 1). Если $\omega(S)<2$, то $\varepsilon(S)=\omega(S)=v(S)$.

ДокАЗАТЕЛЬСТво. По лемме 1 имеем

$$
\omega(S) \leqslant \varepsilon(S) \leqslant v(S)
$$

и поэтому достаточно доказать, что $\omega(S) \geqslant v(S)$. Пусть $\operatorname{dim} E=n$. Из теоремы Пифагора следует, что евклидова норма вектора $S(g) \xi-\xi$ удовлетворяет неравенству

$$
\|S(g) \xi-\xi\|_{2}^{2} \leqslant n(\omega(S))^{2}
$$

для любого (евклидово) единичного вектора $\xi$ в $E$. Поэтому

$$
\|S(g) \xi-\xi\|_{E} \leqslant \sqrt{n} \omega(S)\left(C_{E}\right)^{2}
$$

для любого единичного вектора $\|\xi\|$ (относительно нормы $\|\cdot\|_{E}$ ) в $E$, где

$$
\|\xi\|_{2} \leqslant C_{E}\|\xi\|_{E}, \quad\|\xi\|_{E} \leqslant C_{E}\|\xi\|_{2}
$$

для некоторой постоянной $C_{E}$ и любого вектора $\xi \in E$. Следовательно,

$$
\varepsilon(S) \leqslant \omega(S) \sqrt{n} C_{E} .
$$

Итак, если $S$ - конечномерное представление и $\omega(S)$ конечно, то $\varepsilon(S)$ тоже конечно (см. (4)). Напомним, что представление $S$ локально ограничено по лемме 1. Пусть $M$ - такая постоянная, что $\|S(g)\| \leqslant M$ в некоторой окрестности $U_{0}$ единичного элемента $e$ в $G$. Тогда

$$
\left\|S(g)-I_{E}\right\| \leqslant M+1
$$

где $I_{E}$ - единичный оператор в пространстве $E$.

Пусть $\omega(S)<2$. Докажем, что $v(S) \leqslant \omega(S)$. Пусть $\omega_{0} \in(\omega(S), 2]$ и $\omega_{1} \in$ $\left(\omega(S), \omega_{0}\right)$. Пусть $\delta$ определено условием $0<\delta<\left(\omega_{0}-\omega_{1}\right) /(2(M+1))$. Пусть $\xi_{1}, \ldots, \xi_{p}$ и $f_{1}, \ldots, f_{q}$ - конечные $\delta$-сети на единичных сферах пространства $E$ и пространства $E^{*}$, дуального к $E$, соответственно. Для любых $i$ и $j, i \in\{1, \ldots, p\}$, $j \in\{1, \ldots, q\}$, возьмем такие окрестности $U_{i j}$, что

$$
\left|f_{j}\left(S(g) \xi_{i}-\xi_{i}\right)\right| \leqslant \omega_{1} \quad \text { для любого } g \in U_{i j} .
$$


Пусть

$$
\Omega=U_{0} \cap \bigcap_{\substack{i=1, \ldots, p \\ j=1, \ldots, q}} U_{i j}
$$

Если $\xi \in E,\|\xi\|=1$, и $f \in E^{*},\|f\|=1, g \in \Omega$, и если $i$ и $j$ выбраны так, чтобы

$$
\left\|f-f_{j}\right\| \leqslant \delta, \quad\left\|\xi-\xi_{i}\right\| \leqslant \delta
$$

TO

$$
\begin{aligned}
\|f(S(g) \xi-\xi)\| & \leqslant\left\|\left(f-f_{j}\right)(S(g) \xi-\xi)\right\|+\left\|f_{j}(S(g) \xi-\xi)\right\| \\
& \leqslant \delta(M+1)+\left\|f_{j}\left(\left(S(g)-I_{E}\right)\left(\xi-\xi_{i}\right)\right)\right\|+\left\|f_{j}\left(S(g) \xi_{i}-\xi_{i}\right)\right\| \\
& \leqslant 2 \delta(M+1)+\omega_{1} \leqslant \omega_{0}
\end{aligned}
$$

для всех $\xi \in E,\|\xi\|=1, f \in E^{*},\|f\|=1, g \in \Omega$, и, следовательно,

$$
\left\|S(g)-I_{E}\right\| \leqslant \omega_{0} \quad \text { для любого } g \in \Omega .
$$

Так как $\omega_{0}-$ любое число, бо́льшее $\omega(S)$, то $v(S) \leqslant \omega(S)$, что завершает доказательство леммы.

\section{§3. Критерий непрерывности конечномерных представлений связных компактных групп}

Нам нужно следуюшее вспомогательное утверждение для связных компактных коммутативных групп.

ТЕОРема 4. Пусть $G$ - компактная связная коммутативная группа, в которой есть такой вполне несвязный замкнутый нормальный делитель $N$, что факторгруппа $G / N$ - (компактная коммутативная) группа Ли, т.е. (конечномерный) тор. Пусть $\theta$ - такой характер группь $G$, что

$$
|\theta(g)-1| \leqslant q \quad \text { для некоторого } q<2 \quad \text { и любого } g \in G
$$

в некоторой окрестности $U_{q}$ единицы в группе $G$. Тогда характер $\theta$ непреривен.

ДоКАЗАТЕЛЬСТво. Пусть $T=G / N$ - тор, уПомянутый в условии теоремы. Тогда группа $T$ удовлетворяет всем условиям теоремы 8.20 в [5]. Напомним ее формулировку, что требует некоторых определений. Для любой коммутативной топологической групшы $G$ можно ввести векторное пространство $\mathfrak{L}(G)=\operatorname{Hom}(\mathbb{R}, G)$ однопараметрических групп в $G$. Снабдим это пространство топологией равномерной сходимости на компактах, поточечньм сложением и умножением на числа по правилу

$$
r \cdot X(t)=X(r t) \text { при } X \in \mathfrak{L}(G) \text { и } r, t \in \mathbb{R},
$$

что превращает $\mathfrak{L}(G)$ в топологическую группу векторного пространства; отображение $\exp _{G}: \mathfrak{L}(G) \rightarrow G$, определенное формулой

$$
\exp _{G}(X)=X(1), \quad X \in \mathfrak{L}(G),
$$


называется әкспоненииальной функиией на $G$.

Пусть $\pi: G \rightarrow G / N=T$ - каноническое отображение, где $T$ - топологическая группа, изоморфная тору $\mathbb{T}^{m}$ для некоторого $m \in \mathbb{N}$. По теореме 8.20 в [5] гомоморфизм $\varphi: N \times \mathfrak{L}(T) \rightarrow T$, определенный правилом

$$
\varphi(n, X)=n \exp X, \quad n \in N, \quad X \in \mathfrak{L}(T)
$$

непрерывен, сюръективен и открыт, а его ядро $K$ алгебраически и топологически отображается на подмножество $\Delta=\operatorname{ker}\left(\pi \circ \exp _{T}\right)$ второго сомножителя при проекции $N \times \mathfrak{L}(T) \rightarrow \mathfrak{L}(T)$. Более того, компонента единицы $N \times \mathfrak{L}(T)$ равна $\{0\} \times \mathfrak{L}(T)$, множество $\varphi(\{0\} \times \mathfrak{L}(T))$ плотно в $T$, пересечение $K \cap(\{0\} \times \mathfrak{L}(T))$ имеет вид $\{0\} \times \mathfrak{K}(T)$, где $\mathfrak{K}(T)=\operatorname{ker} \exp _{T}$, и морфизм $\varphi$ факторизуется через факторгруппу $N \times \mathfrak{L}(T) \rightarrow N \times(\mathfrak{L}(T) / \mathfrak{K}(T))$ с фактор-морфизмом $\Phi: N \times(\mathfrak{L}(T) / \mathfrak{K}(T)) \rightarrow T$, заданным форомулой

$$
\Phi(n, X+\mathfrak{K}(T))=n \exp X, \quad n \in N, \quad X \in \mathfrak{L}(T)
$$

Наконец, как было показано в [5] в доказательстве теоремы 8.20, индуцированный морфизм $\mathfrak{L}(\pi): \mathfrak{L}(T) \rightarrow \mathfrak{L}(T / N)$ является изоморфизмом топологических групп векторных пространств, причем экспоненциальные функции $T$ и $T / N$ связаны соотношением

$$
\pi \circ \exp _{T}=\exp _{T / N} \circ \mathfrak{L}(\pi)
$$

В рассматриваемом случае величина $n$ конечна.

Рассмотрим характер $\theta$. По теореме 2 характер групшы Ли $\mathfrak{L}(G)$ (изоморфной $\mathbb{R}^{n}$ ), определяемый композицией $\theta$ ○ $\operatorname{xp}$, непрерывен. Пусть $M-$ компактный открытьй нормальный делитель $N$, лежащий в окрестности $U_{q}[6 ; \S I I .7]$. Тогда $\theta(M)$ - замкнутая подгруппа $\mathbb{T}$, не содержашая некоторой дуги (вблизи точки -1$)$. Поэтому группа $\theta(M)$ конечна. Так как факторгруппа $N / M$ конечна, то и группа $\theta(N)$ конечна. Следовательно, $\theta(N)$ - группа $p$-х корней из единищы для некоторого $p \in \mathbb{N}$. Если $p=1$, то $\theta$ определяется характером групшы Ли $G / N$ и поэтому $\theta$ непрерывен. Пусть $p>1$.

Возьмем $\varepsilon>0$. Пусть $g_{0} \in G$. Так как $G$ связна, то она безгранично делима [5; следствие 8.5]. Поэтому найдется такой элемент $h_{0} \in G$, что $h_{0}^{p}=g_{0}$. Согласно (5) мы можем представить $h_{0}$ в виде $h_{0}=\varphi\left(n_{0}, X_{0}\right)$, т.е. как произведение $h_{0}=n_{0} \exp X_{0}, n_{0} \in N, X_{0} \in \mathfrak{L}(G)$. Возьмем окрестность $U\left(n_{0}\right)$ элемента $n_{0}$ в $N$ и найдем такую окрестность $V\left(X_{0}\right)$ элемента $X_{0}$ в $\mathfrak{L}(G)$, что

$$
\left|(\theta \circ \exp )(X)-(\theta \circ \exp )\left(X_{0}\right)\right|<\varepsilon / p
$$

Так как возведение в $p$-ю степень в группе $G$ есть непрерывный гомоморфизм (поскольку $G$ коммутативна) и притом сюръективньй (поскольку $G$ безгранично делима), то из теоремы об открытом отображении $[6 ; 5.29]$ следует, что это отображение открыто. Так как множество $U\left(n_{0}\right) \exp \left(V\left(X_{0}\right)\right)$ открыто по теореме 8.20 в [5], то множество $W=U\left(n_{0}\right)^{p}\left(\exp \left(V\left(X_{0}\right)\right)^{p}\right)$ есть окрестность точки

$$
n_{0}^{p} \exp \left(X_{0}\right)^{p}=h_{0}^{p}=g_{0}
$$


Если $g \in W$, то $g$ можно представить в виде $g=n^{p}(\exp X)^{p}$ для некоторых $n \in$ $U\left(n_{0}\right)$ и $X \in V\left(X_{0}\right)$. Поэтому

$$
\theta(g)=\theta\left(n^{p}(\exp X)^{p}\right)=(\theta(n))^{p}(\theta(\exp X))^{p} .
$$

Но $\theta(n)^{p}=1$, поскольку порядок группы $\theta(N)$ равен $p$. Таким образом,

$$
\theta(g)=\theta(\exp X)^{p} \quad \text { для любого } g \in W
$$

И

$$
\left|\theta(g)-\theta\left(g_{0}\right)\right|=\left|\theta(\exp X)^{p}-\theta\left(\exp X_{0}\right)^{p}\right| \leqslant p\left|\theta(\exp X)-\theta\left(\exp X_{0}\right)\right|<\varepsilon
$$

по построению, что доказывает непрерьвность характера $\theta$ на $G$.

Следуюший вспомогательный результат также заслуживает выделения в самостоятельное утверждение.

Лемма 3. Пусть $G$ - компактная связная группа и $S$ - (алгебрачческое) представление группы $G$ в конечномерном нормированном линейном пространстве Е. Пусть $\omega(S)<2, \omega_{0}$ - такое число, что $\omega(S)<\omega_{0}<2$, и пусть $\Omega$ - такая окрестность единичного әлемента $e \in G$, что

$$
|f(S(g) \xi-\xi)| \leqslant \omega_{0}\|f\|\|\xi\|, \quad \omega_{0}<2, \quad \text { для любъих } g \in \Omega, \quad \xi \in E, \quad f \in E^{*}
$$

(см. [1; лемма 1]). Пусть $N$ - такой замкнутый нормальный делитель в $G$, что факторгруппа $G / N$ - связная группа Ли [7], [8]. Тогда компонента единицы группы $N$ лежит в ядре представления $S$.

ДокАЗАТЕЛЬСТво. Пусть $\omega_{0}$ удовлетворяет условию $\omega(S)<\omega_{0}<2$. По лемме 1 в [1] существует такая окрестность $\Omega$ единичного элемента $e \in G$, что

$$
|f(S(g) x-x)| \leqslant \omega_{0}\|f\|\|x\|, \quad \omega_{0}<2
$$

для любых $g \in \Omega, x \in E, f \in E^{*}$.

Согласно [7], [8] окрестность $\Omega$ содержит такой замкнутьй нормальный делитель $N$ в $G$, что факторгруппа $G / N$ - (связная) группа Ли. Пусть $N_{0}$ - компонента единицы в $N$.

Напомним, что каждый элемент $g$ произвольной компактной связной топологической группы $H$ содержится в некоторой максимальной компактной связной коммутативной подгруппе $K_{g} \subset H$, любая максимальная связная коммутативная подгруппа в $H$ содержит центр $H$ и любые две связные коммутативные подгруппы в $G$ сопряжены в $H[9]$. Применим это утверждение к компоненте единицы $N_{0}$ группы $N$ (связная группа $N_{0}$ замкнута в $G$ и, следовательно, компактна) и рассмотрим максимальную связную коммутативную подгруппу $L \subset N_{0}$. Замыкание образа этой группы в представлении $S$ - ограниченная (и, следовательно, компактная) коммутативная группа $Q=\overline{S(L)}$ операторов в конечномерном пространстве. Следовательно, группа $Q$ имеет общий собственный базис $f_{1}, \ldots, f_{n}$ в $E$. В этом базисе все 
матрицы, отвечающие операторам из $S(L)$, диагональны и диагональные матричные элементы определяют характеры группы $L$ (они имеют единичный модуль); эти характеры $\chi_{i}: L \rightarrow \mathbb{T}$ определяются правилом

$$
\chi_{i}: l \mapsto s(l)_{i i}, \quad l \in L
$$

Из условия $N_{0} \subset \Omega$ немедленно следует, что образ подгрупшы $N_{0}$ под действием любого диагонального характера $\chi_{i}=s_{i i}$ не содержит некоторую дугу окружности $\mathbb{T}$ вблизи точки $-1 \in \mathbb{T}$ (размер этой дуги определяется значением разности $2-\varepsilon>0)$. Таким образом, образ $s_{i i}(L)$ есть либо единичная подгруппа, либо группа $q$-х корней из единицы для некоторого натурального $q \geqslant 2$. Однако второй случай невозможен, потому что группа $L$ связна по построению и, следовательно, безгранично делима [5; следствие 8.5], в то время как любой гомоморфный образ безгранично делимой групшы тоже безгранично делим. Итак, $S$-образ $L$ есть $\{1\}$. Так как любой элемент группы $N_{0}$ сопряжен элементу группы $L$, то $S\left(N_{0}\right)=\{1\}$.

Теперь мы можем получить критерий непрерывности конечномерных представлений связных компактных групп.

ТЕОРема 5. Пусть $G$ - компактная связная группа и $S$ - (алгебраическое) представление группь $G$ в конечномерном нормированном линейном пространстве E. Если $\omega(S)<2$, то $v(S)=0$, т.е. представление $S$ непрерьвно.

ДокАЗАТЕЛЬСтво. Примем обозначения и определения, использованные в лемме 3. По этой лемме имеем $S\left(N_{0}\right)=\{1\}$. Поэтому мы можем рассматривать представление $S$ групшы $G$ как представление компактной связной факторгрупшы $G / N_{0}$. Группа $N / N_{0}$ - вполне несвязный нормальньй делитель в связной групе $G / N_{0}$. Следовательно, группа $N / N_{0}$ центральна в $G / N_{0}$.

Применим утверждение о максимальных связных коммутативных подгруппах к группе $G / N$. Пусть $T^{\prime}$ - максимальная связная коммутативная подгруппа $G / N$. Образ $S\left(T^{\prime}\right)$ ограничения представления $S$ на подгруппу $T^{\prime}$ есть ограниченная коммутативная группа операторов в конечномерном пространстве. Следовательно, эта группа имеет общий собственный базис $x_{1}, \ldots, x_{n}$ в $E$. В этом базисе матрицы из семейства $S\left(T^{\prime}\right)$ диагональны, и диагональные матричные элементы определяют характеры групшы $T^{\prime}$, имеющие единичный модуль; эти характеры $\chi_{i}: T^{\prime} \rightarrow \mathbb{T}$ определяются формулами

$$
\chi_{i}: l \mapsto s(l)_{i i}, \quad l \in T^{\prime}
$$

По построению каждый характер вида $\chi_{i}$ удовлетворяет условиям теоремы 4 (окрестность, в которой значения характера не попадают на дугу вблизи точки -1 , есть пересечение канонического образа исходной окрестности в $G$ в групе $G / N_{0}$ с максимальной связной коммутативной подгруппой $T^{\prime}$ ). Следовательно, все характеры $\chi_{i}$ непрерывны и ограничение представления $S$ на $T^{\prime}$ непрерывно. Так как $T^{\prime} \supset N / N_{0}$, то ограничение представления $S$ на $N / N_{0}$ непрерывно. Так как $N / N_{0}$ вполне несвязна, то сушествует такой открытый нормальный делитель $M \subset N / N_{0}$, что $S(g)=I_{E}$ для всех $g \in M$. Тогда нормальный делитель $Q=\left.\operatorname{ker} S\right|_{N / N_{0}}$ содержит $N / N_{0}$ и поэтому тоже открыт. Пусть $P$ - полный 
прообраз $Q$ при каноническом отображении $G$ на $G / N_{0}$. Тогда $P$ - открытый нормальный делитель в $N$. Рассмотрим $S$ снова как представление группы $G$. Тогда $P=\operatorname{ker} S \cap N$. Следовательно, $P$ - компактный открытый нормальный делитель в $G$. Поэтому индекс $P$ в $G$ конечен. Кроме того, $P$ содержит $N_{0}$. Поэтому индекс $\operatorname{ker} S \cap N$ в $N$ конечен. Таким образом, $G /(\operatorname{ker} S \cap N)-($ связная) группа Ли и представление $S$ можно рассматривать как представление этой группы Ли. Кроме того, представление $S$, рассматриваемое как представление группы Ли $G /(\operatorname{ker} S \cap N)$, имеет то же значение $\omega(S)$, так что $\omega(S)<2$. Остается применить теорему 2 , что завершает доказательство.

\section{§4. Критерий непрерывности конечномерных представлений связных разрешимых групп}

Нам придется сначала доказать аналог теоремы Ли для конечномерных представлений разрешимых групп.

ТЕОРема 6. Пусть $G$ - разрешимая связная локально компактная группа и $S$ - (алгебраическое) представление группы $G$ в конечномерном нормированном линейном пространстве $E$. Если $\omega(S)<2$, mо $v(S)=0$, т.е. представление $S$ непрерывно.

ДокАЗАТЕЛЬСтво. Докажем теорему индукцией по длине композиционного ряда разрешимой связной локально компактной групшы $G$. Если эта длина равна единице, то группа $G$ коммутативна. Согласно части (iii) теоремы 7.57 в [5] связная коммутативная локально компактная группа есть (алгебраически и топологически) прямое произведение конечномерной векторной группы $V$ и (единственной) максимальной компактной подгрупшы $K$. Заметим, что группа $K$ связна как гомоморфный образ связной групшы $G$. В этом случае утверждение теоремы 6 следует из теоремы 5 , примененной к группе $K$, и теоремы 2 , примененной к группе $V$. Таким образом, база индукции установлена.

Докажем утверждение индукционного шага. Пусть утверждение справедливо, если длина композиционного ряда разрешимой связной локально компактной группы не превосходит натурального числа $n$, и пусть дана разрешимая связная локально компактная группа $G$, длина композиционного ряда которой равна $n+1$. Пусть $S$ - (алгебраическое) представление групшы $G$ в конечномерном нормированном линейном пространстве $E$, и пусть $\omega(S)<2$. Рассмотрим коммутаторную подгруппу $C$ группы $G$, т.е. замкнутую подгруппу, порожденную коммутаторами

$$
g h g^{-1} h^{-1}, \quad g, h \in G \text {. }
$$

Тогда $H$ - разрешимая локально компактная группа, длина композиционного ряда которой равна $n$, и $C$ связна как замыкание связного множества (объединения связных множеств, образованных элементами, допускающими представление в виде произведения данного числа коммутаторов; эти множества имеют общую точку (единицу группы )). Пусть $T$ - ограничение представления $S$ на подгруппу $C$. Очевидно, что $\omega(T)<2$. По предположению индукции представление $T$ групшы $C$ непрерывно. Следовательно, по теореме Ли [10; теорема V.3.1] представление $T$ 
имеет одномерное инвариантное подпространство (и тем самьм общий собственньй вектор $\xi \in E)$ :

$$
T\left(g h g^{-1} h^{-1}\right) \xi=\lambda(g, h) \xi, \quad \lambda(g, h) \in \mathbb{C}, \quad g, h \in G .
$$

Тогда

$$
\begin{gathered}
T\left(g h g^{-1} h^{-1}\right) S(k) \xi=S(k) T\left(k^{-1} g h g^{-1} h^{-1} k\right) \xi=\lambda\left(k^{-1} g k, k^{-1} h k\right) S(k) \xi \\
g, h, k \in G
\end{gathered}
$$

так что все векторы вида $S(k) \xi$ - собственные для $T$. Они порождают конечномерное подпространство $F \subset E$ (линейную оболочку одномерных $T$-инвариантных подпространств в $E$ ), и подпространство $F$ имеет базис из собственных векторов представления $T$. Разумеется, множество линейно независимых векторов вида $T(k) \xi, k \in G$, конечно. Из формулы (6) следует, что подпространство $F$ инвариантно относительно представления $S$ групшы $G$. Обозначим через $S_{F}$ ограничение представления $S$ на подпространство $F$. Таким образом, множество значений функции $\lambda$ конечно. Отметим также, что (при фиксированных $g, h \in G$ ) элемент $k^{-1} g h g^{-1} h^{-1} k$ принадлежит $C$, поскольку имеет место равенство

$$
\begin{aligned}
k^{-1} g h g^{-1} h^{-1} k & =\left(k^{-1} g k\right)\left(k^{-1} h k\right)\left(k^{-1} g^{-1} k\right)\left(k^{-1} h^{-1} k\right) \\
& =\left(k^{-1} g k\right)\left(k^{-1} h k\right)\left(k^{-1} g k\right)^{-1}\left(k^{-1} h k\right)^{-1},
\end{aligned}
$$

и непрерывно зависит от $k \in G$. Кроме того, функция $\lambda$ непрерывна, поскольку представление $T$ непрерывно. Так как группа $G$ связна, то функция $G \rightarrow \mathbb{C}$, определенная формулой

$$
k \mapsto \lambda\left(k^{-1} g k, k^{-1} h k\right), \quad k \in G,
$$

постоянна, т.е. не зависит от $k \in G$. Следовательно, оператор $T\left(g h g^{-1} h^{-1}\right)$ скалярен на $F$ (где он является умножением на число $\lambda(g, h))$. Но

$$
T\left(g h g^{-1} h^{-1}\right)=S_{F}(g) S_{F}(h) S_{F}\left(g^{-1}\right) S_{F}\left(h^{-1}\right) \quad \text { для любых } g, h \in G
$$

и из $S$-инвариантности подпространства $F$ следует, что

$$
\operatorname{det} T\left(g h g^{-1} h^{-1}\right)=1, \quad(\lambda(g, h))^{\operatorname{dim} F}=1
$$

Таким образом, множество значений функции $\lambda$ конечно. Из связности групшы $G$ следует тогда, что множество значений функции $\lambda$ одноэлементно. Так как число 1 заведомо входит в это множество значений, то

$$
\lambda(g, h) \equiv 1 \quad \text { для всех } g, h \in G \text {. }
$$

Следовательно, представление $T$ кратно единичному, $C \subset \operatorname{ker} T$ и можно считать, что $S_{T}$ - представление факторгруппы $G / C$. Обозначим через $S_{F}^{G / C}$ ограничение представления $S$ на подпространство $F$, рассматриваемое как представление факторгрупгы $G / C$. Так как группа $G / C$ коммутативна и связна, а $\omega\left(S_{F}^{G / C}\right)<2$ по 
условию и по определению топологии в факторгруппе, то представление $S_{F}^{G / C}$ непрерывно по уже доказанному утверждению, составляющему базу индукции. Таким образом, данное представление имеет инвариантное подпространство и ограничение на это подпространство является непрерывным представлением.

Переходя к факторпространству $E / F$ и повторяя рассуждение, мы получим по индукции, что можно выбрать базис в пространстве представления $S$, в котором матрицы представления имеют блочно-треугольный вид с непрерывньми диагональными блоками.

Рассмотрим пространство $\mathscr{L}(E)$ всех линейных операторов в пространстве $E$ представления $S$. Пусть $M$ - пересечение замыканий $\overline{S(V)}$ образов $S(V)$ всевозможных окрестностей $V \subset G$ единичного элемента группы $G$. Поскольку представление $S$ ограничено на некоторой окрестности $V_{0}$ единичного элемента, то для любой окрестности $V \subset V_{0}$ множество $\overline{S(V)}$ компактно как замкнутое подмножество компактного множества.

Пусть $T$ - некоторьй элемент $M$. Равенство $M=\left\{1_{E}\right\}$ равносильно непрерывности представления $S$ в $е$ ввиду только что отмеченной компактности множеств $\overline{S(V)}$ в $\mathscr{L}(E)$. Действительно, дополнение любой окрестности нуля $W$ в множестве $\overline{S\left(V_{0}\right)}$ (для такой окрестности $V_{0}$, что $\overline{S\left(V_{0}\right)}$ компактно) тоже компактно. Если $M=\left\{1_{E}\right\}$, то пересечение $M \cap\left(\overline{S\left(V_{0}\right)} \backslash W\right)$ пусто и поэтому существует конечньй набор множеств вида $\overline{S\left(V_{i}\right)}, i=1, \ldots, N$, для которых пересечение

$$
\left(\bigcap_{i=1}^{N} \overline{S\left(V_{i}\right)}\right) \cap\left(\overline{S\left(V_{0}\right)} \backslash W\right)
$$

пусто, так что

$$
S(V) \subset W \quad \text { для } \quad V=V_{0} \cap\left(\bigcap_{i=1}^{N} V_{i}\right) .
$$

Докажем, что $T=1_{E}$. Так как для любого единичного вектора $\xi \in E$ и любого функционала $f \in E_{*}$ единичной длины окрестность $U(\xi)$ участвует в построении множества $M$, то $|f(T \xi-\xi)|<2$ для всех единичных векторов $\xi \in E$ и функционалов $f \in E_{*}$ единичной длины. Таким образом, $\left\|T-1_{E}\right\| \leqslant 2$.

Для любой окрестности $V$ единичного элемента в $G$ и любого натурального $n$ существует окрестность $W$ единичного элемента в $G$, удовлетворяющая условию $W^{2^{n}} \subset V$. Отсюда следует, что $S(W)^{2^{n}} \subset S(V)$ и, очевидно, $(S(W))^{2^{n}} \subset S(V) \subset$ $\overline{S(V)}$. Пусть $n=1$. Тогда $S(W) S(W) \subset S(V)$ для любого $\alpha$ и так как умножение операторов непрерывно, то $\overline{S(W) S(W)} \subset \overline{S(V)}$. По индукции отсюда следует, что для любой окрестности $V$ единичного элемента в $G$, любого натурального $n$ и любой окрестности $W$ единичного элемента в $G$, удовлетворяющей условию $W^{2^{n}} \subset V$, имеем $(\overline{S(W)})^{2^{n}} \subset \overline{S(V)}$ в $\mathscr{L}(E)$. В частности, поскольку $T \in \overline{S O}$ для любой окрестности $O$ единичного элемента, то $T^{n} \in \overline{S(V)}$ для всех $n \in \mathbb{N}$ и для всех окрестностей $V$ единичного элемента в $G$, откуда следует, что $T^{n} \in M$. Как мы видели выше, отсюда следует, что

$$
\left\|T^{n}-1_{E}\right\| \leqslant 2 \text { для всех } n \in \mathbb{N} .
$$

Напомним, что в пространстве представления $S$ есть базис, в котором матрицы представления имеют блочно-треугольный вид с непрерывньми диагональными 
блоками. В этом базисе оператор $T$ имеет блочно-треугольньй вид с единичными диагональными блоками. Если $T \neq 1_{E}$, то последовательность $T^{n}$ неограничена, что противоречит неравенству (7). Поэтому представление $S$ непрерывно в единичном элементе, а тогда и всюду на $G$. Это доказьвает теорему 6.

\section{§5. Критерий непрерывности конечномерных представлений связных локально компактных групп}

Теперь мы можем доказать основную теорему статьи.

ТЕОРемА 7. Пусть $G$ - связная локально компактная группа и $\sigma$ - (алгебраическое) представление группь $G$ в конечномерном нормированном линейном пространстве Е. Если $\omega(\sigma)<2$, то $v(\sigma)=0$, т.е. представление $\sigma$ непрерывно.

ДоКАЗАТЕЛЬСТво. Как и в доказательстве теоремы 5 , примем обозначения и определения, использованные в лемме 3 . По этой лемме имеем $S\left(N_{0}\right)=\{1\}$. Поэтому мы можем рассматривать представление $\sigma$ группы $G$ как представление $\sigma^{\prime}$ связной факторгрупшы $G^{\prime}=G / N_{0}$. Очевидно, что выполняется условие $\omega\left(\sigma^{\prime}\right)<2$. Поэтому мы перейдем к $G^{\prime}=G / N_{0}$ и $\sigma^{\prime}$, не будем писать штрихи и будем считать, что $N_{0}=\{e\}$. Этот переход позволяет считать, что группа $N$ - вполне несвязный нормальньй делитель в связной группе $G$, причем факторгруппа $G / N$ есть связная группа Ли. Отсюда следует, что группа $N$ центральна в $G$, а $G$ - конечномерная группа. Пусть $\pi: G \rightarrow \Sigma$ - каноническое отображение. Рассмотрим радикал (наибольший связный разрешимьй нормальньй делитель) группы $G$ (см. [11; теорема 3.7]). Тогда либо $G=R$, либо факторгруппа $\Sigma=G / R$ есть связная полупростая локально компактная группа конечной размерности [12; теорема 7.6]. Рассмотрим образ $\pi(Z(G))$ центра $Z(G)$ группы $G$ в группе $\Sigma$. Так как $\pi$ - эпиморфизм, то $\pi(Z(G))$ содержится в центре $Z(\Sigma)$ групшы $\Sigma$. В этом случае $Z(G) \supset N$ и поэтому группа $\Sigma / Z(\Sigma)$ есть связная полупростая группа Ли без центра (прямое произведение присоединенных групп простых групп Ли).

Если сама группа $\Sigma$ - группа Ли, то ее центр конечен. Следовательно, есть замкнутая подгруппа $Q$ конечного индекса в $Z(G)(Q$ - полный прообраз единицы в $Z(\Sigma))$, лежащая в $R$.

Пусть $\Sigma$ - не группа Ли. Напомним [12; теорема 8.3], что в этом случае сушествует последовательность

$$
\pi_{n}: L_{n+1} \rightarrow L_{n}, \quad n \in \mathbb{N}
$$

нетривиальных конечнократных накрытий связных групп Ли, где $L_{0}=\Sigma / Z(\Sigma)$ (прямое произведение присоединенных групп простых групп Ли) и $\Sigma$ - проективный предел семейства $\left(L_{n}\right)_{n \in \mathbb{N}}$, где каждая группа $L_{n}$ есть гомоморфный образ универсальной накрьвающей групшы $L_{0}$, которая изоморфна прямому произведению универсальных накрывающих простых сомножителей групшы $L_{0}$. Напомним, что для простого сомножителя в $\Sigma / Z(\Sigma)$ последовательность $\left\{\pi_{n}\right\}$ может сушествовать тогда и только тогда, когда этот сомножитель эрмитов. В этом случае центр любой группы $L_{n}$ содержится в центральном торе максимальной компактной подгруппы группы $L_{n}$. Поскольку неэрмитовы прямые сомножители в $\Sigma / Z(\Sigma)$ 
имеют универсальные накрывающие группы с конечньм центром, то центр $Z(\Sigma)$ группы $\Sigma$ содержит подгруппу $Z^{\prime}$ конечного индекса (ядро факторизации группы $\Sigma$ по прообразу в $\Sigma$ прямого произведения всех неэрмитовых прямых сомножителей в $\Sigma / Z(\Sigma)$ ), лежащую в проективном пределе прямого произведения одномерных центральных торов максимальных компактных подгрупп эрмитовых сомножителей $\left[12 ; 8.5\right.$ и теорема 8.6]. Тогда группа $Z^{\prime}$ лежит в проективном пределе $T^{\prime}$ этих прямых произведений. Группа $T^{\prime}$ связна и коммутативна как проективный предел торов. Ее полный прообраз $R_{1}$ в $G$ разрешим как расширение связной коммутативной групшы $T^{\prime}$ с помощью связной разрешимой группы $R$, и группа $R_{1}$ содержит центральную подгруппу $Q$ конечного индекса в $Z(G)$.

Итак, в любом случае центр $Z(G)$ группы $G$ содержит замкнутую подгруппу $Q$ конечного индекса, принадлежащую замкнутой связной разрешимой подгруппе $R_{1}$ группы $G$. По теореме 6 ограничение представления $\sigma$ групшы $G$ на подгруппу $R_{1}$ непрерывно. Следовательно, ограничение представления $\sigma$ групшы $G$ на подгруппу $Q$ непрерывно. Поскольку $Q$ - подгруппа конечного индекса в $Z(G)$, то ограничение представления $\sigma$ группы $G$ на подгруппу $Z(G)$ непрерьвно, откуда следует, что замкнутый нормальньй делитель $\operatorname{ker} \sigma \cap Z(G)$ имеет конечный индекс в $Z(G)$. Таким образом, представление $\sigma$ можно рассматривать как представление связной факторгруппы $G /(\operatorname{ker} \sigma \cap Z(G))$. Эта группа есть расширение группы Ли $G / Z(G)$ с помощью конечной группы $Z(G) /(\operatorname{ker} \sigma \cap Z(G))$ и потому является группой Ли. Остается применить теорему 2.

\section{§6. Примеры и заключительные замечания}

6.1. Наилучшие константы. Приведем примеры, показывающие, в частности, что постоянная 1 в теореме 1 и постоянные 2 и $\sqrt{3}$ в теоремах 2 и 3 неулучшаемы.

ПримеР 1. Пусть $G$-недискретная локально компактная группа, $\Sigma$-представление групшы $G$ в гильбертовом пространстве $l^{2}(G)$ сдвигами. Тогда $\omega(\Sigma)=1$ и при этом представление $\Sigma$ разрывно.

Пример 2. Пусть $G$ - недискретная локально компактная группа, $E$ - замыкание конечных линейных комбинаций характеристических функций точек в банаховом пространстве ограниченных функций на $G$ с обычной равномерной нормой, $\Sigma-$ представление группы $G$ в $E$ сдвигами. Тогда $\varepsilon(\Sigma)=1$ и при этом представление $\Sigma$ разрывно.

ПримеР 3. Пусть $G=\mathbb{T}, \Sigma$ - нетривиальный одномерный характер группы $G$, все неединичные значения которого имеют бесконечный порядок (см., например, [6; $(25.26)($ e)]. Тогда $\omega(\Sigma)=\varepsilon(\Sigma)=2$ и при этом представление $\Sigma$ разрывно.

ПримеР 4. Пусть $G$ - прямое произведение счетного набора групп третьего порядка, реализованных в виде корней из единицы на комплексной плоскости,

$$
\mathbb{T}_{3}=\{1, \cos (2 \pi / 3) \pm \sin (2 \pi / 3)\}
$$

занумеруем эти групшы натуральными числами. Рассмотрим некоторый свободный ультрафильтр $\alpha$ на натуральном ряде и введем одномерный характер $\Sigma$ группы $G$ с помошью формулы

$$
\Sigma(g)=\alpha\left(\left(g_{n}\right)\right) \text { при } g=\left(g_{n}\right) \in G,
$$


где $g_{n} \in \mathbb{T}_{3}$ для всех $n \in \mathbb{N}$. Тогда $\omega(\Sigma)=\varepsilon(\Sigma)=2$ и при этом представление $\Sigma$ разрывно.

Проверка правильности вычисления величин $\omega(\Sigma)$ и $\varepsilon(\Sigma)$ и проверка разрывности представлений $\Sigma$ в этих примерах предоставляется читателю.

6.2. Приложение: непрерывность представлений, аппроксимирующих конечномерные квазипредставления.

СлЕДСТвИЕ. Пусть $G$ - связная локально компактная группа и $T$ - ее непрерывное отображение в группу обратимых линейных операторов в конечномерном пространстве Е. Если существует такое представление $\Sigma$ группь $G$ в $E$, что $\|\Sigma(g)-T(g)\| \leqslant C$ для всех $g \in G$ и некоторого $C, C<1$, то представление $\Sigma$ непрерывно.

ДокАЗАТЕЛЬСтво. В Условиях следствия имеем $\omega(\Sigma) \leqslant \omega(T)+2 C$, где $\omega(T)=0$ по условию непрерывности отображения $T$. Следовательно, $\omega(\Sigma)<2$. Остается применить теорему 7.

\section{3. Условия непрерывности конечномерных представлений общих локально компактных групп.}

В случае общих (не обязательно связных) локально компактных групп ситуация оказывается более содержательной.

ТЕОРема 8. Все конечномернье представления открытой почти связной подгруппь $O$ локально компактной группь $G$, колебание которых в единице группы меньше 2 , непрерывны в том и только том случае, если все нормальнъе делители нечетного индекса в любой открытой подгруппе некоторой открытой почти связной подгруппы $\sigma$ группы $G$ замкнуты.

Группा $O$ и $\sigma$ могут быть различными.

Доказательство теоремы 8 , которое будет опубликовано в другой статье, существенно использует условия непрерьвности конечномерных представлений вполне несвязных локально компактных групп (см. [4]).

Автор благодарен А.С. Мишенко за внимание к работе.

\section{Список литературы}

1. IIтерн А.И. Критерии слабой и сильной непрерьвности представлений топологических групп в банаховых пространствах // Матем. сб. 2002. Т. 193. № 9. С. 139-156.

2. Shtern A. I. Continuity of Banach representations in terms of point variations // Russian J. Math. Phys. 2002. V. 9. № 2. P. 251-253.

3. Shtern A. I. Continuity criteria for finite-dimensional representations of compact connected groups // Adv. Stud. Contemp. Math. 2003. V. 6. № 2. P. 141-156.

4. Shtern A. I. Continuity conditions for finite-dimensional representations of some compact totally disconnected groups // Adv. Stud. Contemp. Math. 2004 (to appear).

5. Hofmann K. H., Morris S. A. The structure of compact groups. Berlin: Walter de Gruyter, 1998.

6. Хьюитm Э., Росс К. А. Абстрактний гармонический анализ. Т. І. М.: Наука, 1975.

7. Montgomery D., Zippin L. Topological transformation groups. New York: Interscience Publ., 1955.

8. Глушков B. M. Структура локально бикомпактных групп и пятая проблема Гильберта // УМH. 1957. T. 12. № 2. С. 3-41. 
9. Ahdout S., Hurwitz C., Itzkowitz G., Rothman S., Strassberg H. Maximal protori in compact topological groups // Papers on general topology and applications (Flushing, NY, 1992). New York: New York Acad. Sci., 1994. P. 227-236. (Ann. New York Acad. Sci. V. 728.)

10. Наймарк М. А. Теория представлений групп. М.: Наука, 1976.

11. Paterson A. L. T. Amenability. Providence, RI: Amer. Math. Soc., 1988. (Math. Surveys Monogr. V. 29.)

12. Stroppel M. Lie theory for non-Lie groups // J. Lie Theory. 1994. V. 4. № 2. P. 257-284.

Московский государственнй университет

им. М.В. Ломоносова

E-mail: ashtern@member.ams.org
Поступила в редакцию 6.10 .2003 